Article

\title{
Isolation and Molecular Characterization of Thirteen R2R3-MYB Transcription Factors from Epimedium sagittatum
}

\author{
Wenjun Huang ${ }^{1, \dagger}$,Wei Sun ${ }^{2, \dagger}$, Haiyan Lv ${ }^{1}$, Gong Xiao ${ }^{1}$, Shaohua Zeng ${ }^{2}$ and Ying Wang ${ }^{1, *}$
}

1 Key Laboratory of Plant Germplasm Enhancement and Specialty Agriculture,

Wuhan Botanical Garden, Chinese Academy of Sciences, Wuhan 430074, Hubei, China;

E-Mails: wjhuang@wbgcas.cn (W.H.); hyl@wbgcas.cn (H.L.); gongxiaobio@gmail.com (G.X.)

2 Key Laboratory of Plant Resources Conservation and Sustainable Utilization, South China Botanical Garden, Chinese Academy of Sciences, Guangzhou 510650, Guangdong, China; E-Mails: djsunwei@gmail.com (W.S.); shhzeng@scbg.ac.cn (S.Z.)

$\dagger$ These authors contributed equally to this work.

* Author to whom correspondence should be addressed; E-Mail: yingwang@wbgcas.cn; Tel.: +86-27-8751-0675; Fax: +86-27-8751-0331.

Received: 16 November 2012; in revised form: 11 December 2012 / Accepted: 11 December 2012 / Published: 27 December 2012

\begin{abstract}
Epimedium sagittatum (Sieb. et Zucc.) Maxim, a popular traditional Chinese medicinal plant, has been widely used for treating sexual dysfunction and osteoporosis in China. The main bioactive components in herba epimedii are prenylated flavonol glycosides, which are end products of a branch of the flavonoid biosynthetic pathway. The MYB transcription factors (TF) act as activators or repressors to regulate the flavonoid pathway. In this study, 13 full-length cDNA clones of $R 2 R 3-M Y B$ TFs from E. sagittatum (designated as $E S M Y B 1$ to $E S M Y B 13$ ) were isolated and characterized. Sequence similarity and phylogenetic analysis placed nine $R 2 R 3-M Y B$ members of E. sagittatum into five subgroups of the Arabidopsis R2R3-MYB family, while four members were not clustered into a defined subgroup. The number and length of introns from Epimedium $R 2 R 3-M Y B$ genes varied significantly, but intron positions and phases were well conserved. Expression patterns of Epimedium $R 2 R 3-M Y B$ genes in various tissues showed diverse. Finally, it is suggested that five Epimedium $R 2 R 3-M Y B$ genes may be involved in regulating the flavonoid pathway and could be used as valuable candidate genes for metabolic engineering studies in future. Sequence information of $13 R 2 R 3-M Y B$ genes discovered
\end{abstract}


here will also provide an entry point into the overview of whole $R 2 R 3-M Y B$ family in Epimedium.

Keywords: Epimedium; medicinal plant; flavonoid pathway; MYB; transcription factor

\section{Introduction}

Transcription factors (TFs) play a central role in developmental and metabolic programs by regulating the transcription expression of downstream target genes. The MYB proteins comprise one of the largest TF families in the plant kingdom [1,2]. MYB proteins have two distinct regions, an $N$-terminal conserved MYB DNA-binding domain (MYB domain) and a diverse $C$-terminal modulator region that is responsible for the regulatory activity of the protein. The MYB domain is highly conserved among plants, yeast and animals [3], and its consensus sequence contains approximately 50 amino acid residues with regularly spaced tryptophan forming a helix-turn-helix structure [4]. Based on the number of MYB domain, the MYB family can be divided into four classes, 1R-, R2R3-, 3R- and 4R-MYB proteins [2,5]. R2R3-MYB proteins are specific to plants and are also the most abundant type in plants, with greater than $100 R 2 R 3-M Y B$ members in the genomes of dicots and monocots [5-8]. Plant $R 2 R 3-M Y B$ proteins play an important role in many biological processes including primary and secondary metabolism, cell fate and identity, developmental processes and responses to biotic and abiotic stress $[5,9,10]$.

Since the first gene $(C l)$ encoding a plant MYB protein was identified in maize [11], a tremendous number of plant MYB genes have been characterized, of which many $R 2 R 3-M Y B$ genes were demonstrated to regulate the phenylpropanoid and flavonoid biosynthetic pathway in several plant species, including Arabidopsis, apple, grapevine, maize, petunia and snapdragon [12]. In Arabidopsis for example, AtTT2 regulates proanthocyanin (PA) biosynthesis in the seed coat [13], while AtPAP1 and $A t P A P 2$ activate the phenylpropanoid biosynthetic genes and enhance anthocyanin accumulation in vegetative tissues [14]. In addition, grapevine $V v M Y B P A 1, V v M Y B A 1, V v M Y B 5 a$ and $V v M Y B F 1$ controls PA, anthocyanin, phenylpropanoid and flavonol biosynthesis, respectively [9,15-17]. It is well established that MYB TFs interact with bHLH TFs, which act together with WD40 protein to regulate the flavonoid biosynthetic pathway and cell fate [18-20].

Herba epimedii, one of the most popular traditional Chinese medicines, is collected from the dried aerial parts of Epimedium species (Berberidaceae family), widely distributed in China [21]. Herba epimedii has extensive pharmacological efficacy for treating sexual dysfunction and infertility, preventing cardiovascular diseases, protecting against osteoporosis, strengthening immune system and improving whole body health as well as possessing anti-oxidation, anti-tumor, and anti-aging effects [22,23]. The herb E. sagittatum (Sieb. et Zucc.) Maxim recorded in the Chinese Pharmacopoeia [24] contains high level of bioactive compounds and is widely used as a Chinese herbal medicine. In addition, Epimedium species are used as ground covers and ornamental plants due to the abundance of colors and patterns in their leaves and flowers [25].

It has been reported that the main constituents of Epimedium species which contribute to various bioactivities are prenylated flavonol glycosides [22,26], end-products of a branch of the flavonoid 
biosynthetic pathway. To date, more than 260 compounds have been isolated and identified in the genus Epimedium, among which C-8 prenylated flavonol glycosides are the major compounds, and four of these (epimedin A, B, C and icariin) are used as important bioactive markers for quality control and chemotaxonomic classification [22,27]. However, the molecular biosynthesis and regulatory mechanism to produce these bioactive components is still unclear. The recent development of an EST database from E. sagittatum will accelerate the molecular isolation of genes involved in the flavonoid pathway [25].

Wild natural resources of medicinal Epimedium species have declined dramatically in recent times due to years of over-harvesting and habitat destruction and these species have now become endangered [28]. Considering the shortage of natural resources and the large growing market demand for natural medicines, it is essential to better understand the biosynthesis and regulation of bioactive compounds in high-quality germplasm resources, in order to enable development of new cultivars with the improved traits for large-scale cultivation.

The $R 2 R 3-M Y B$ family has been divided into several subgroups [5,29], and the functions of $R 2 R 3-M Y B$ proteins from the same subgroup are broadly conserved in different angiosperms [2]. Thus, it may be predicted that several MYB proteins could regulate the flavonoid biosynthetic pathway and other secondary metabolite pathways involved in the biosynthesis of bioactive compounds in E. sagittatum. However, no data has been available for $R 2 R 3-M Y B$ genes in the genus Epimedium to date. This study also is thus the first to analyze the genomic structure of the $R 2 R 3-M Y B$ genes in E. sagittatum and their phylogenetic relationships with other plant $M Y B$ genes. In short, we aim to isolate and characterize several members of $R 2 R 3-M Y B$ family that are proposed to play an important role in controlling the flavonoid biosynthetic pathway to pave the way for genetic manipulation of bioactive compound biosynthesis in Epimedium species. Specifically, we isolated and characterized 13 different $R 2 R 3-M Y B$ full-length sequences from $E$. sagittatum. The full-length coding sequences enabled us to explore their phylogenetic relationships with other plant $M Y B$ genes and further predict their biological functions. We also analyzed the genomic structures of these isolated $R 2 R 3-M Y B$ genes to obtain insights into their evolution. The mRNA expression patterns of these $M Y B$ genes were examined by quantitative RT-PCR (qRT-PCR) assay in various tissues. At last, we found that five $R 2 R 3-M Y B$ genes are likely to regulate the flavonoid biosynthetic pathway in Epimedium.

\section{Results}

\subsection{Isolation and Sequence Analysis of 13 R2R3-MYB Genes from Epimedium}

A total of 13 full-length cDNA clones of R2R3-MYBTFs were isolated from leaves of E. sagittatum using homology-based cloning, RACE (rapid amplification of cDNA ends) technology and fishing for candidate ESTs encoding MYB proteins in the Epimedium EST database [25]. Each of the R2R3-MYB cDNA clones contained one whole open reading frame (ORF), varying from 735 bp (EsMYB5) to $1323 \mathrm{bp}$ (ESMYB2) in length and encoding 244 to 440 amino acids (aa) with an average length of about 310 aa (Table S1). All Epimedium R2R3-MYB sequences contained the R2 and R3 MYB domain identified through conserved domain analysis, placing them in the large $R 2 R 3-M Y B$ family (Figure 1a). In most of EsMYB members, the R2 and R3 MYB domains were arranged in tandem as is typical for 
plant $R 2 R 3-M Y B$ proteins, but a fragment of approximate 50 aa separated the $\mathrm{R} 2$ and $\mathrm{R} 3 \mathrm{MYB}$ domain of EsMYB8 (Figure 1b). Similarly to other plant $R 2 R 3-M Y B$ proteins, all EsMYB proteins were highly conserved in the $N$-terminal region and highly variable in the $C$-terminal region. Alignment of the deduced amino acid sequences for $12 E s M Y B$ genes, excluding the atypical EsMYB8, revealed an overall protein identity varying from $5 \%$ to $66 \%$, while the MYB domain represented a high level of amino acid conservation (44\%-90\% identities), particularly in the R3 repeat domain (Figure 1a). Five pairs of EsMYB proteins with very high identities $(>80 \%)$ in their MYB domains were found: EsMYB3 and EsMYB13, EsMYB7 and EsMYB10, EsMYB2 and EsMYB6, EsMYB1 and EsMYB12, and EsMYB5 and EsMYB11 (Figure 1a).

Figure 1. Sequence alignment of $12 R 2 R 3-M Y B$ proteins within the MYB domains from E. sagittatum except EsMYB8 and their R2 and R3 MYB domains arrangement. (a) Identical residues are shown in black and similar residues in gray. The R2 and R3 MYB domains are underlined. The two arrowheads indicate the intron I and intron II insertion site, respectively. (b) The R2 and R3 MYB domains are arranged in tandem in 12 of 13 EsMYB proteins, except EsMYB8 protein in which a fragment of about 50 amino acid spaces the R2 and R3 repeat domain.

(a)

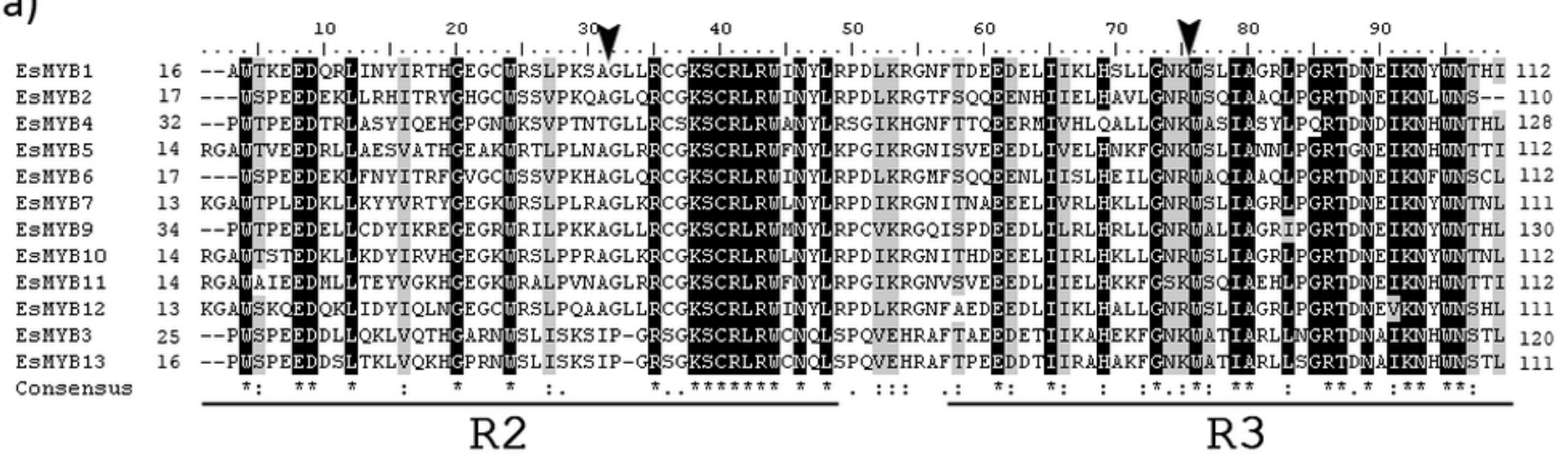

(b)

MYB

Protein

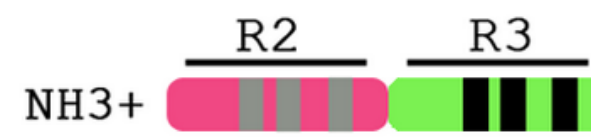

$\mathrm{COO}-$

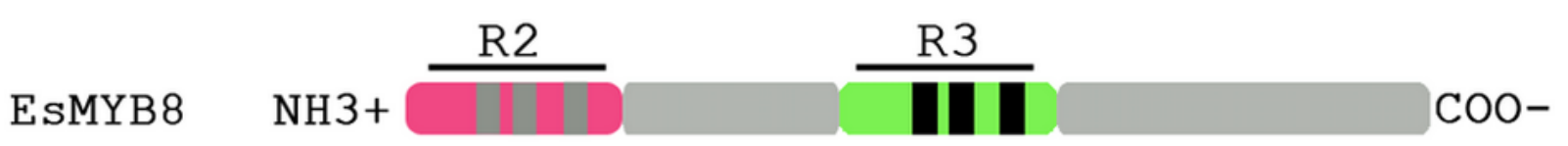

A blast search of all EsMYB genes in the Arabidopsis nr database revealed that each EsMYB gene possessed many corresponding homologues from Arabidopsis with high identity (Table S2). For example, EsMYB7 and EsMYB10 showed high homology with the Arabidopsis AtTT2 (AtMYB123) gene involved in regulating proanthocyanin (PA) accumulation in the seed coat [13], while EsMYB1 and ESMYB12 were highly homologous with the transcriptional repressor AtMYB4, which negatively regulates the expression of cinnamate 4-hydroxylase [30] (Table S2). In addition, blast analysis of Epimedium MYB genes in the nr database excluding Arabidopsis revealed that EsMYB5 and EsMYB11 shared approximately 50\% identity with $V v M Y B P A 2$ which regulates PA biosynthesis in grape [31], 
and $E S M Y B 9$ shared $56 \%$ identity with grape $V v M Y B 5 b$ which regulates the flavonoid pathway during fruit development [9] (Table S3), and EsMYB8 was highly similar to AtMYB (At5g58900) and other plant DIV (DIVARICATA) and DIV-like genes (e.g., AmDIV, BIDIV, HmDIV and ZmDIV) involved in the dorsoventral asymmetry of flowers [32,33] (Table S3).

To gain further insight into the predicted functions of $M Y B$ genes from Epimedium, additional sequence analysis was performed. The bHLH interacting motif ([D/E]Lx2[R/K]x3Lx6Lx3R) in the R3 domain [34] was present in five Epimedium R2R3-MYB genes, including EsMYB1, EsMYB7, EsMYB9, $E S M Y B 10$ and $E S M Y B 12$ (data not shown). Each ESMYB gene clustered into one subgroup of the Arabidopsis R2R3-MYB family possessed the subgroup-specific conserved motifs located outside the MYB domain [5,29]. EsMYB7 and ESMYB10 shared a similar motif with the R2R3-MYB subgroup 5-specific conserved motif (DExWRLxxT) [5], while EsMYB1 and EsMYB12 contained two $R 2 R 3-M Y B$ subgroup 4-specific conserved motifs: $\mathrm{C} 1$ (LlsrGIDPxT/SHRxI/L) and C2 (pdLNLD/ELxiG/S), of which the $\mathrm{C} 2$ motif forms part of the region involved in transcriptional repression [30]. Unlike other members of subgroup 4, however ESMYB12 did not contain a Zinc-finger motif $\left(\mathrm{Cx}_{1-2} \mathrm{Cx}_{7-12} \mathrm{Cx}_{2} \mathrm{C}\right)$ in the $C$-terminus [29], while EsMYB1 did (Figure S1). Moreover, EsMYB9 contained the $\mathrm{C} 1$ motif of $R 2 R 3-M Y B$ subgroup 4 [29] as well as the $\mathrm{C} 3$ motif (DDxF[S/P]SFL[N/D]SLIN[E/D]) identified previously in $V v M Y B 5 a$ and $V v M Y B 5 b$ clusters $[9,35]$, but not the $\mathrm{C} 2$ motif [30] (Figure S2). Unlike typical plant $R 2 R 3-M Y B$ genes, ESMYB8 has longer linkers (about 55 aa) between the R2 and R3 repeat domains and a specific "VASHAQKYF" motif sequence located towards the end of the R3 MYB domain, and another conserved motif followed closely in the $C$-terminus was also found in the EsMYB8 sequence (Figure S3) [36].

\subsection{Phylogenetic Relationships and Genomic Structure Analysis of Epimedium R2R3-MYB Genes}

A phylogenetic tree of 13 complete $R 2 R 3-M Y B$ proteins from E. sagittatum and 166 MYB proteins downloaded from the Arabidopsis Plant Transcription Factor Database was constructed using the neighbor-joining method. Nine $E S M Y B$ members clustered into five previously defined subgroups (SG) of the R2R3-MYB family in Arabidopsis, including SG1, SG4, SG5, SG13 and SG22 [5,29], while four $E S M Y B$ genes (ESMYB5, ESMYB8, EsMYB9 and ESMYB11) did not fall into a defined subgroup (Figure 2). In accordance with their SG-specific motifs, ESMYB1 and EsMYB12 were clustered into SG4, EsMYB7 and EsMYB10 into the SG5; while EsMYB9 showed a close relationship with AtMYB5, but it was not assigned to a defined subgroup (Figure 2). The topology tree indicated that most of EsMYB proteins were placed at the basal position of a group or branch (Figure 2).

Phylogenetic analysis of $13 R 2 R 3-M Y B$ genes from $E$. sagittatum with other functionally characterized plant $M Y B$ genes was also conducted to further predict their potential biological functions. The result was in agreement with that of the phylogenetic tree of Epimedium and Arabidopsis $M Y B$ sequences (Figure S4). ESMYB1 showed a close relationship with the AtMYB4 and $A m M Y B 308$ transcriptional repressors [30,37], while ESMYB12 was clustered closely with the FaMYB1 repressor from Fragaria x ananassa with a high bootstrap value [38] (Figure S4). EsMYB9 clustered into the $V v M Y B 5 a$ and $V v M Y B 5 b$ group identified previously [9,35], and EsMYB7 and ESMYB10 were clustered with TT2 homologues from Arabidopsis and Lotus, but the bootstrap value 
was relatively low (Figure S4) [13,39]. EsMYB8 clustered with DIV homologues from Aquilegia coerulea with a high bootstrap value (Figure S4).

Figure 2. Phylogenetic relationships of $13 R 2 R 3-M Y B$ genes from E. sagittatum and 166 MYB gene models from Arabidopsis thaliana. Phylogenetic tree is constructed using the neighbor-joining method by the MEGA 4.0 version [40]. $13 R 2 R 3-M Y B$ genes from Epimedium are shown in diamond. All $166 M Y B$ gene models are downloaded from the Arabidopsis thaliana MYB Transcription Factor database [41] and their gene model accession numbers are shown. Arabidopsis R2R3-MYB subgroups are designated as previously reported [5,29]. The putative functions of the different $M Y B$ genes in the control of secondary metabolite biosynthesis or other biological processes are indicated.

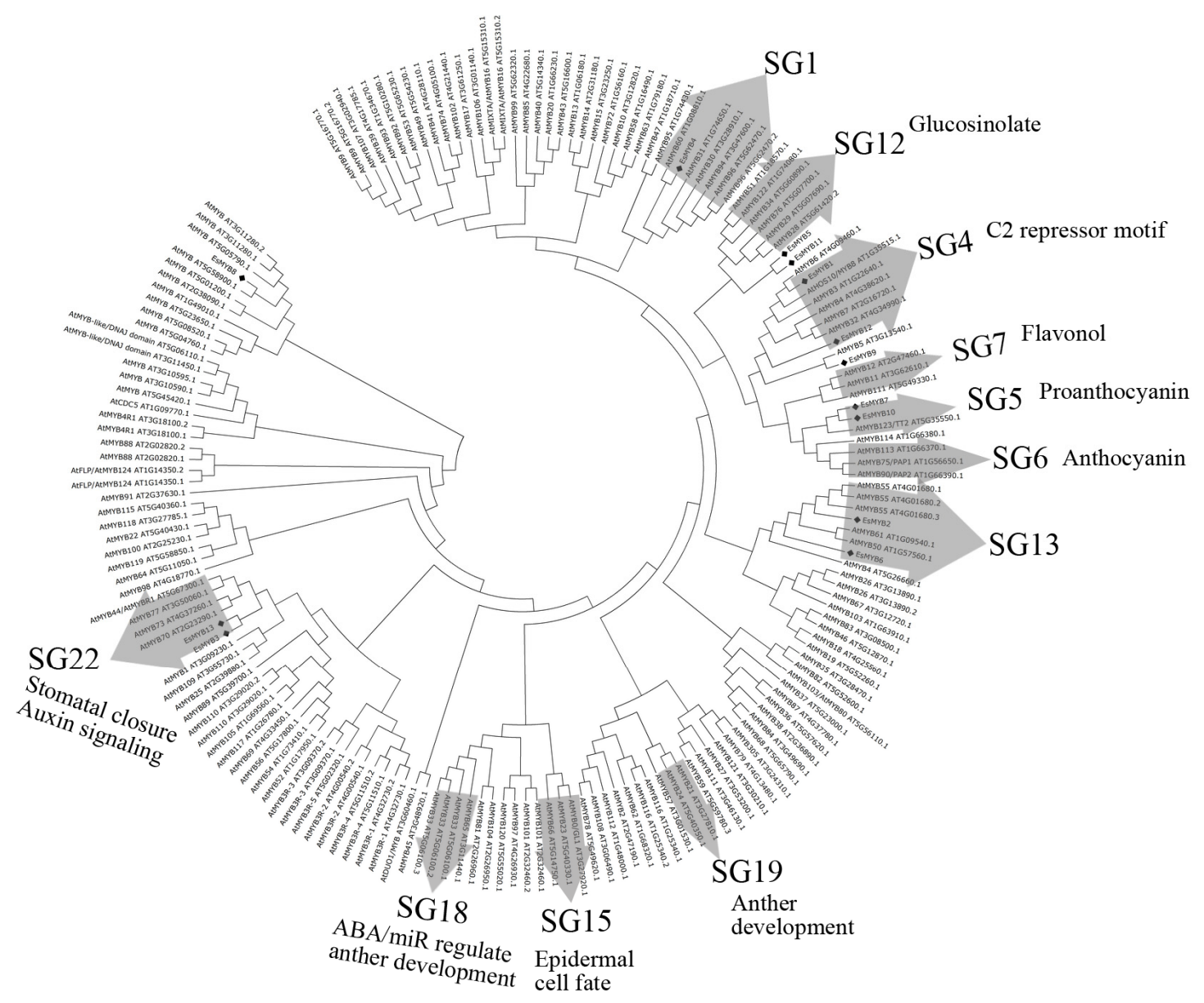

The genomic structure of Epimedium $R 2 R 3-M Y B$ genes was analyzed by pair-wise alignment of their full-length coding sequences and corresponding genomic DNA (gDNA) sequences. Three types of exon/intron structure were found in the all 13 R2R3-MYB genes and designated as Class I, II and III. Class I contained three exons and two introns (nine members); Class II, two exons and one intron (EsMYB8 and EsMYB9); Class III, one exon and no intron (EsMYB3 and EsMYB13) (Figure 3). To further verify the Class III genomic structure and exclude possible DNA contamination in the cDNA isolation, both the full-length cDNA and gDNA clones of ESMYB3 and EsMYB13 from cDNA template digested with DNase I and genomic DNA template were amplified and compared at the same time. The band size was as same as expected between the cDNA and gDNA clone of each gene 
(Figure S5). The average length of both intron I and intron II was found to be same (125 bp), but ranging from $83 \mathrm{bp}$ (ESMYB10) to $272 \mathrm{bp}$ (EsMYB7) for intron I, and from 66 (EsMYB11) to $280 \mathrm{bp}$ (ESMYB8) for intron II, respectively (Table S1). The splice junction sites of all $M Y B$ genes were conserved for GT and AG dinucleotide, except for ESMYB1 intron II where the acceptor site was TG (Table S1). Moreover, the intron positions of 13 R2R3-MYB genes exception EsMYB8 were conserved, which intron I and intron II inserted between the conserved A and G residues of the R2 MYB domain and $(\mathrm{R} / \mathrm{K}) \mathrm{W}$ residues of the R3 MYB domain, respectively (Figure 1a). The intron II insertion site of ESMYB 8 was between $\mathrm{K}_{134}$ and $\mathrm{L}_{135}$, which is consistent with the insertion positions and phases from other plant $D I V$ genes (Figure S3) [36].

Figure 3. Genomic structures of $13 R 2 R 3-M Y B$ genes from E. sagittatum. Exons and introns are shown in boxes and lines, respectively. The numbers at the left and right side indicate the position of the translation start codon and stop codon, respectively. The scale represents $100 \mathrm{bp}$ length in nucleotide. Detailed information about exon and intron length and splice junction site is seen in Table S1.

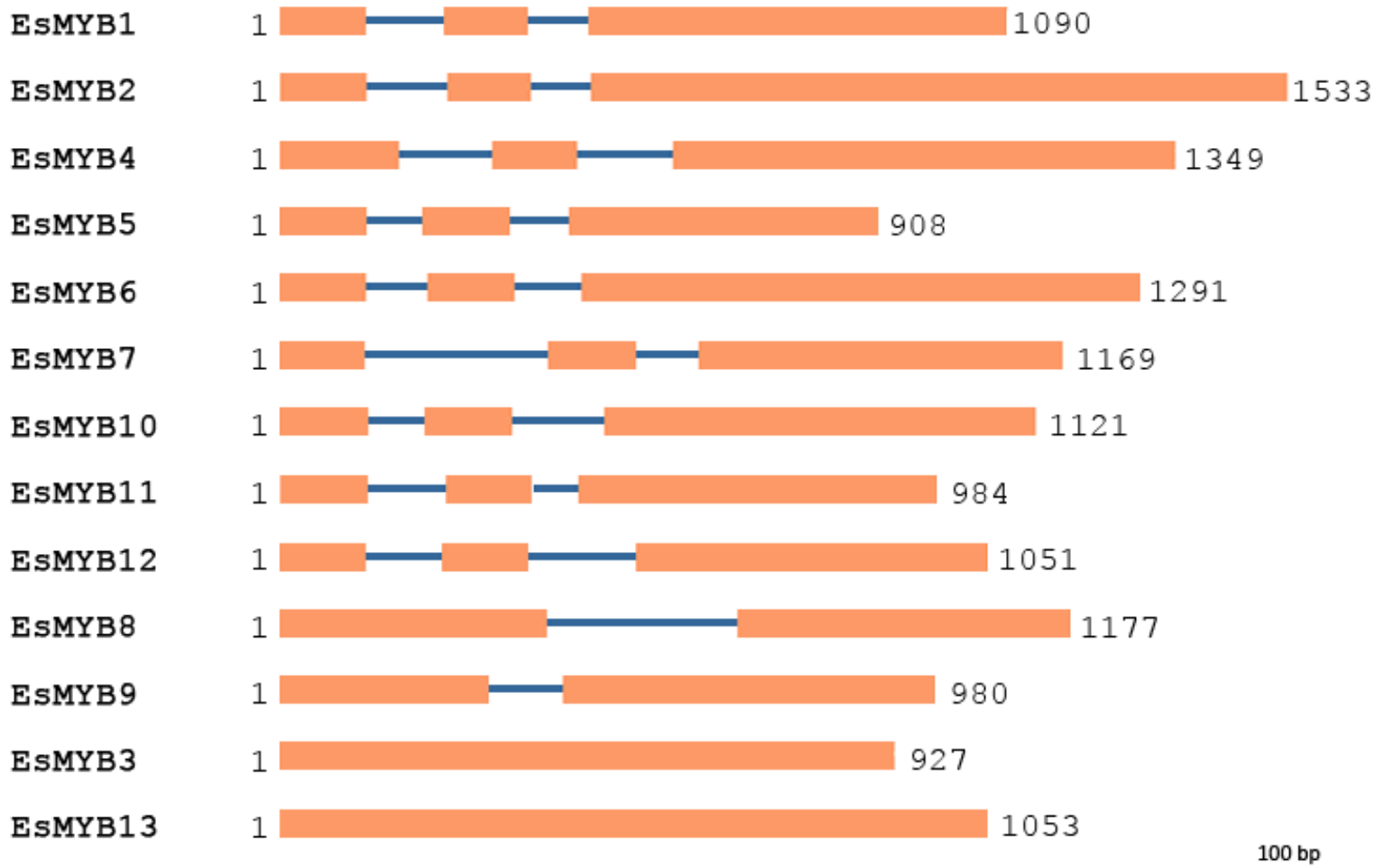

\subsection{Expression Patterns of Epimedium R2R3-MYB Genes in Various Tissues}

Expression patterns of 13 cloned $R 2 R 3-M Y B$ genes from E. sagittatum were investigated by quantitative RT-PCR (qRT-PCR) in various tissues, including young leaf, young petiole, flower, fruit and root (Figure 4a). The results showed that seven EsMYB genes were expressed in all tissues tested, with highest abundance in different tissues for each gene, and some ESMYB genes were expressed preferentially in the specific tissues, with highest expression in root for EsMYB1, EsMYB6, EsMYB8 and $E S M Y B 13$. In addition, six $E S M Y B$ genes showed low or no transcripts in fruit tissue, and five $E S M Y B$ genes were most highly expressed in the young leaf (Figure 4a). Specifically, the mRNA level 
of EsMYB9 was highest in the flower, and absent in fruit and root tissues. EsMYB1 and EsMYB12 showed different expression patterns, with ESMYB1 expressed in all tissues and most highly in the root, while ESMYB12 was expressed in leaf, petiole and flower tissues at the similar level. EsMYB7 was most abundantly expressed in the fruit and detected in all tissues, while EsMYB10 was abundantly expressed in leaf and flower tissues, but lowly in fruit and root tissues (Figure 4a).

Figure 4. Expression patterns of $13 R 2 R 3-M Y B$ genes from E. sagittatum in various tissues. (a) The mRNA expression patterns of 13 Epimedium $R 2 R 3-M Y B$ genes in leaf, petiole, flower, fruit and root tissues are examined by qPCR assay. The comparative $\mathrm{Ct}$ method is used to determine the relative expression, and the expression level of gene in leaf tissue is set to "1". (b) Five Epimedium R2R3-MYB genes (ESMYB1, EsMYB7, EsMYB9, $E S M Y B 10$ and $E S M Y B 12)$ are selected to determine their mRNA expression levels by qPCR assay (right) in the red and green leaf tissues (left) which accumulate different amount of anthocyanin (middle). Three samples are used from two plants for qPCR assay, and the red young leaf and green old leaf are collected from the same plantlet at differential developmental stages, while the green young leaf is collected from another plantlet at the same developmental stage of the red young leaf. Bar $=1 \mathrm{~cm}$. Total anthocyanin content is determined using spectrophotometric method, and the column represents the mean value and SD (standard deviation) indicating from three replicates. The comparative $\mathrm{Ct}$ method is used to determine the relative expression level, and the expression level of gene in green old leaf is set to " 1 ".

(a)

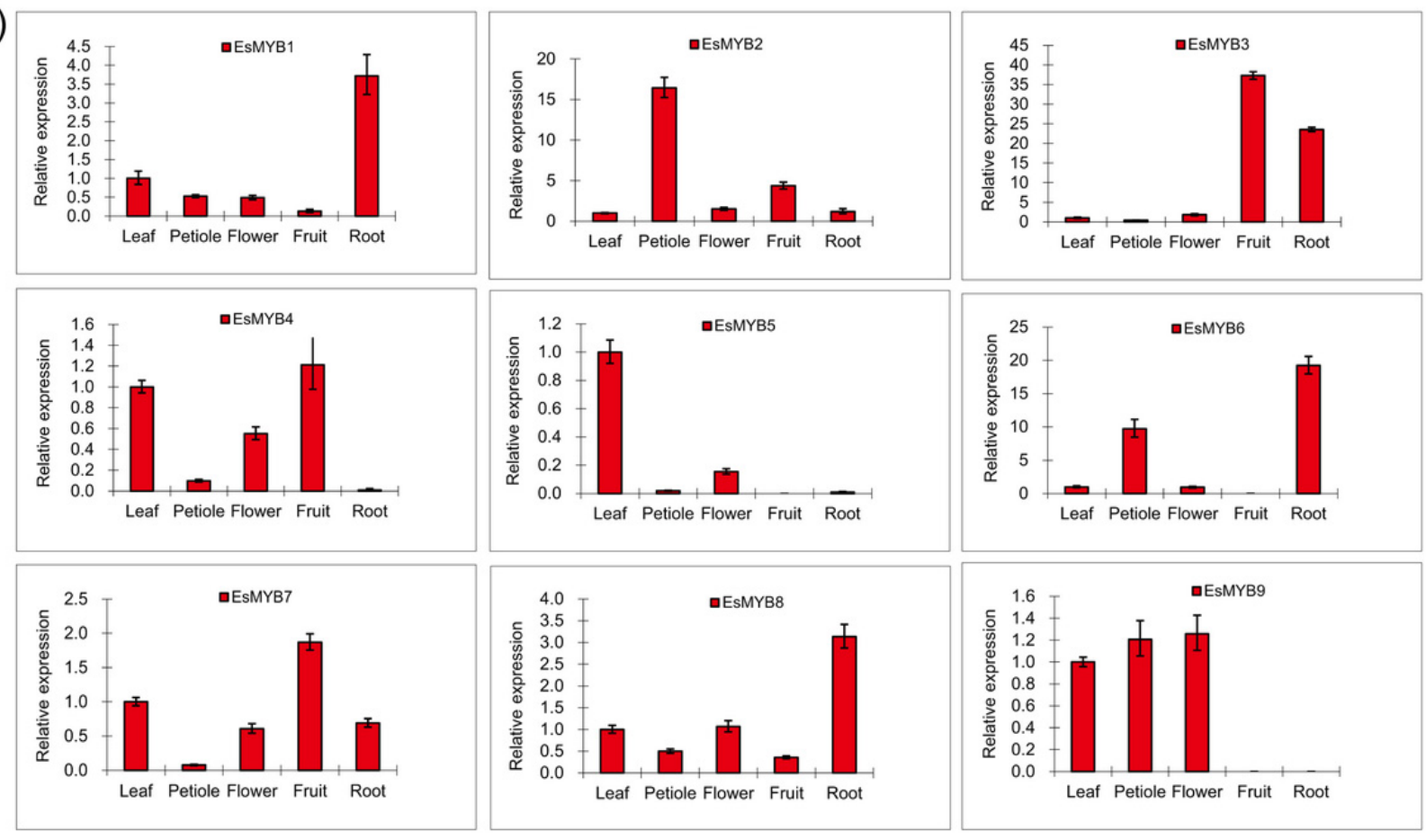


Figure 4. Cont.

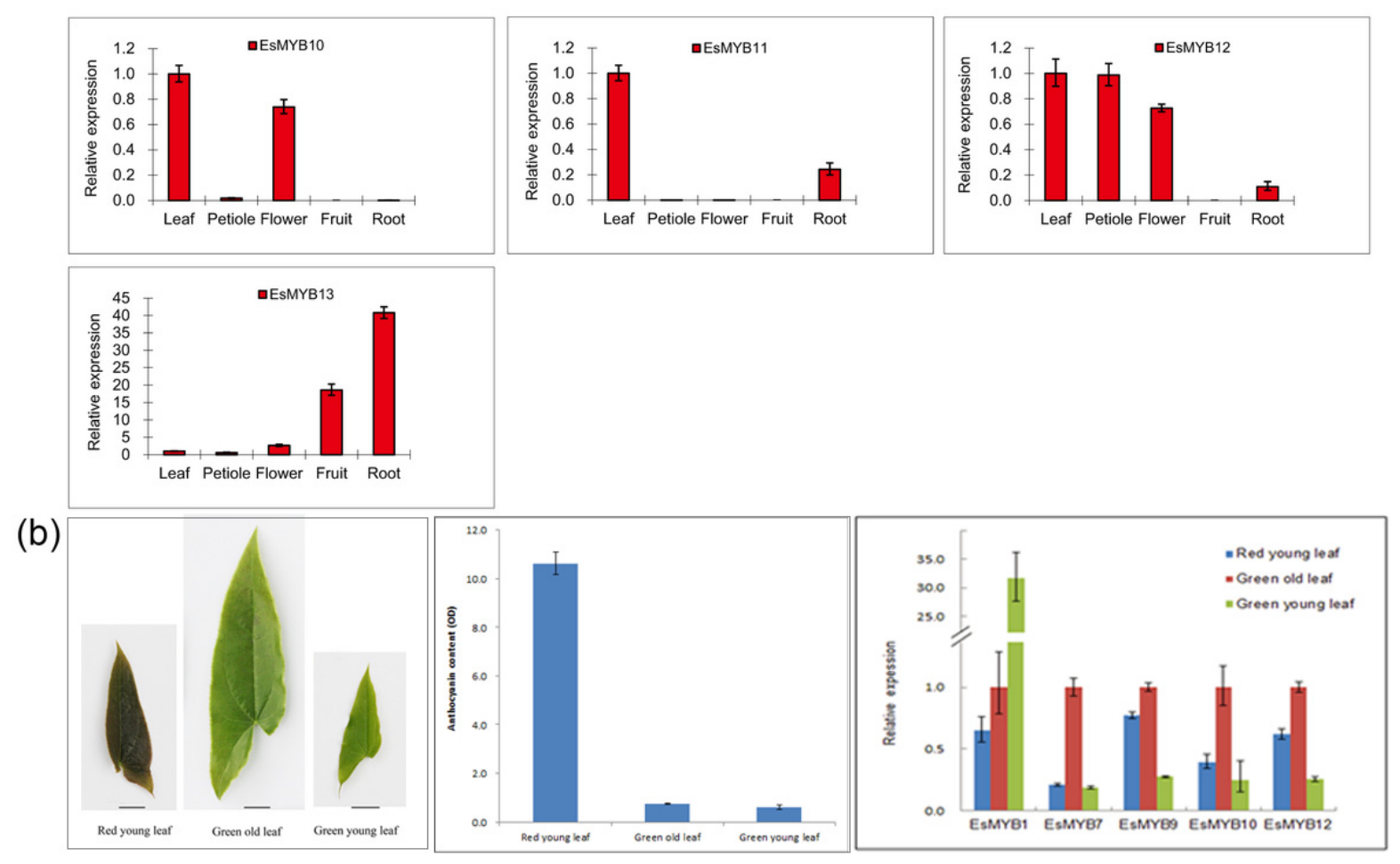

In order to infer the functions of some $E S M Y B$ genes further, the mRNA abundances of five selected $E S M Y B$ (EsMYB1, EsMYB7, EsMYB9, EsMYB10 and EsMYB12) were compared by qRT-PCR in red versus green leaves, which accumulate different anthocyanin contents (Figure 4b). A total of three leaves samples were collected for qRT-PCR analysis, with sample 1 and 2 corresponding to the red young leaf and green old leaf from the same plantlet but collected at different developmental stages, and sample 3 corresponding to the green young leaf collected from another plantlet at the same developmental stage of the sample 1. The results indicated that the expression levels of all five EsMYB genes were higher in the green old leaf (sample 2) than in the red young leaf (sample 1) from the same plant, and $E S M Y B 9, E S M Y B 10$ and $E S M Y B 12$ genes were more abundantly expressed in the red young leaf (sample 1) than in the green young leaf (sample 3). EsMYB1, on the other hand, was approximately 48 times higher in the green young leaf (sample 3) than in red young leaf (sample 1) (Figure 4b).

\section{Discussion}

In this study, the complete coding sequences and intron-exon structures of 13 Epimedium genes that share the characteristic features of $R 2 R 3-M Y B$ family were reported. The phylogenetic relationships of Epimedium $R 2 R 3-M Y B$ genes with other plant $M Y B$ genes were examined and their mRNA expression patterns in several tissues were also examined to predict their potential functions.

Our data revealed that the MYB domains of Epimedium $R 2 R 3-M Y B$ genes are highly conserved, whereas the $C$-terminal regions are highly variable, as shown in earlier studies of other plant $M Y B$ genes [2]. The conserved motif for interaction with $b H L H$ TFs, which is usually present in the R3 repeat domain of $R 2 R 3-M Y B$ regulators controlling the proanthocyanin (PA) and anthocyanin 
biosynthetic pathway [34,42], was found in five Epimedium $R 2 R 3-M Y B$ genes. The Arabidopsis $R 2 R 3-M Y B$ family has been divided into 22 subgroups, and nine of thirteen Epimedium $R 2 R 3-M Y B$ genes were dispersed into five subgroups and thus contained the subgroup-specific conserved motifs [5,29].

Amino acid motifs of several subgroups are broadly conserved among different species [8,29,43]. ESMYB1 and ESMYB12 located in subgroup 4 (SG4) shared the C2 repressor motif (pdLNLD/ELxiG/S), which plays a key role in transcription repression [30]. Besides the C2 repressor motif, all members of SG4 homologues, including ESMYB1 and ESMYB12, harbored a conserved bHLH interaction motif [34], suggesting ESMYB1 and ESMYB12 could interact with $b H L H$ TF. The overall structural homology between EsMYB1, EsMYB12 and other C2 repressor-containing MYB proteins from different plant species, and the reports that several of them act as transcriptional repressors of the phenylpropanoid pathway suggest that EsMYB1 and EsMYB12 may function as transcriptional repressors of the flavonoid pathway in Epimedium. It is noticeable that EsMYB1 shared a Zn-finger motif with most of members of SG4, but EsMYB12 did not. However, it is reported that the strawberry $F a M Y B 1$ which acts as a transcription repressor of the anthocyanin and flavonol pathway also contains the $\mathrm{C} 2$ repressor motif but no Zn-finger motif [38]. Moreover, EsMYB 12 showed a closer relationship with FaMYB1 and had a different mRNA expression pattern with EsMYB1 which clustered with AtMYB4 and AmMYB308, suggesting divergent tissue-specific activity of these genes.

It is proposed that $M Y B S$ generally regulate only one branch of the flavonoid pathway [12]. MYB regulators of PA biosynthesis have been identified in several plant species, including AtTT2 from Arabidopsis, LjTT2 from Lotus japonicus, VvMYBPA1 and VvMYBPA2 from Vitis vinifera [13,15,31,39]. EsMYB7 and EsMYB10 had high identity with AtTT2 homologues and were clustered into SG5, and showed close relationships with $M Y B$ regulators controlling the PA biosynthesis. Although neither protein contained the conserved motif $[\mathrm{V}(\mathrm{I} / \mathrm{V}) \mathrm{R}(\mathrm{T} / \mathrm{P})(\mathrm{K} / \mathrm{R}) \mathrm{A}(\mathrm{I} / \mathrm{L} / \mathrm{V})(\mathrm{R} / \mathrm{K}) \mathrm{C}]$ present in the highly variable $C$-terminal region of both AtTT2 and $O S M Y B 3[5,13]$, this 9-residue consensus sequence was also not found in the amino acid sequence of LjTT2 controlling PA biosynthesis in Lotus [39]. The MYB domains of EsMYB7 and EsMYB10 shared greatest identity with GhMYB10 from Gossypium hirsutum and a high similarity of $81 \%-87 \%$ with the LjTT2 genes. Thus, it is predicted here that ESMYB7 and ESMYB10 are possibly the MYB regulators of PA biosynthesis in Epimedium. On the other hand, EsMYB5 and ESMYB11 showed similarity with $V v M Y B P A 1$, but they were not clustered together and lacked the $b H L H$ interaction motif, which is necessary for the $M Y B$ regulators of PA biosynthesis. Thus, it is inferred that EsMYB5 and ESMYB11 are not true candidate regulators of PA biosynthesis in Epimedium.

Sequence similarity of MYB proteins is mainly confined to the R2 and R3 MYB domains, however MYB proteins can share conserved motifs in their $C$-terminal regions and this may indicate similarities in function [5]. ESMYB9 showed high identity with $V v M Y B 5 a$ and $V v M Y B 5 b$ genes of grape and fell into a small clade with five other $M Y B$ genes involved in the control of various physiological and developmental processes [9,35]. Like the $V v M Y B 5 a$ and $V v M Y B 5 b$ cluster, EsMYB9 contained the C1 motif initially described in the $R 2 R 3-M Y B$ SG4 proteins and the $\mathrm{C} 3$ motif only present in the all members of the $V v M Y B 5 a$ and $V v M Y B 5 b$ small cluster. This, and the fact that transgenic tobacco lines overexpressing both ESMYB9 and the $V v M Y B 5 a$ and $V v M Y B 5 b$ genes showed similar phenotypes [9,35] indicates functional conservation of these orthologs in different species. 
Specifically, for $E s M Y B 9$ overexpression transgenic tobacco lines, significant changes in coloring were showed in petals of transgenic flowers and in stamens where the amount of pigments was clearly greater (Figure S6). In the vegetative tissues of transgenic tobacco lines no visible phenotypic differences were observed, compared to wild-type plants (data not showed).

Exon/intron structures are highly conserved between $R 2 R 3-M Y B$ family members in Epimedium and several model species. Four groups of $R 2 R 3-M Y B$ family have been classified in the grape and Arabidopsis based on the split position of the R2 and R3 repeats within the MYB domain [8]. Three groups are found in the Epimedium $R 2 R 3-M Y B$ members, just lacking the group II that only has the R2 repeat split between exon 1 and 2. The fact that just over a dozen Epimedium MYB sequences are available for analysis may provide a reliable explanation. In nine of thirteen Epimedium $R 2 R 3-M Y B$ genes, exon 1 and 2 encode for almost the entire R2 and R3 MYB domain, and are restricted in length (about $130 \mathrm{bp}$ ), as reported in Arabidopsis and grape [8]. In addition, exon 3 of EsMYB genes encodes for the last region of the $\mathrm{R} 3$ repeat and for the $C$-terminal region of the protein, and is the most diverse in size. Nine of thirteen (69\%) of Epimedium $R 2 R 3-M Y B$ genes consisted of 3 exons and 2 introns, which is consistent with the reports that a majority of Arabidopsis (59\%) and rice (53\%) MYB genes had this exon/intron structure [6]. It is noticeable that the complete ESMYB3 and ESMYB13 proteins were encoded by a single exon, which has not been seen to date in grape [8]. Although the size of introns varied greatly for different $E S M Y B$ genes, the intron positions and phases were well conserved. EsMYB8 had only one intron, which split the R3 repeat domain at a distinctive position, but it was highly conserved with the closely related DIVARICATA (DIV) genes from other plants [36]. Although the sequence information of 13 Epimedium $R 2 R 3-M Y B$ genes is limited, it may provide an insight into the genomic structure of whole Epimedium $R 2 R 3-M Y B$ family.

Different $R 2 R 3-M Y B$ genes from Epimedium had different expression patterns. EsMYBI and $E S M Y B 12$ are suggested here to act as transcriptional repressors, and showed different expression profiles. EsMYBIwas most highly expressed in roots, which is similar to that seen for EgMYB1 of Eucalyptus gunnii preferentially expressed in differentiating secondary xylem of the stems and roots [44]. Moreover, it was observed that EsMYBI was higher in the green leaves than in the red leaves which accumulate more anthocyanin contents. The preferential accumulation of EsMYB1 in lignin-rich tissue suggests a possible role in the regulation of lignin biosynthesis. Unlike EsMYB1, $E S M Y B 12$ was abundantly expressed in leaf, petiole and flower tissues which accumulate a certain amount of anthocyanins. ESMYB12was not expressed in fruit, which is contrary to the reports about the closely related FaMYB1 repressor of strawberry. FaMYB1 showed the highest expression level in the red ripe strawberry fruit and repressed transcription to balance the levels of anthocyanin at the latter stages of fruit maturation [38]. The high expression levels of ESMYB12 in anthocyanin-rich tissues perhaps suggest that EsMYB12 is involved in the regulation of anthocyanin biosynthesis. In addition, the fact that ESMYB12 transcript increased during the maturation stage of leaves and was higher in red young leaves than in green young leaves is counterpart of the FaMYB1 expression profile [38]. Aside from the transcriptional repressors, EsMYB9 and both EsMYB7 and EsMYB10 are possibly transcriptional activators of the flavonoid and PA biosynthetic pathways, respectively. EsMYB9was abundantly expressed in the anthocyanin-rich tissues, which is partly consistent with the high expression of $V v M Y B 5 a$ and $V v M Y B 5 b$ in leaves and berries of grape [9,35]. Moreover, EsMYB9 transcript was higher in old leaves than in young leaves, as shown for $V v M Y B 5 b$ [9]. Given that 
ESMYB9 is suggested to be a $M Y B$ regulator of the flavonoid pathway, it is surprising that no transcript was found in fruit and roots. The EsMYB7 expression was not limited to certain tissues, which coincides with the expression patterns of LjTT2 genes [39]. It is reported that the expression levels of TT2 homologues correlated with the PA accumulation patterns [13,39]. PA is accumulated in several organs and tissues for Lotus japonicus, whereas PA accumulation in Arabidopsis is limited to the seed coat, which indicates the different species-specific expression patterns of TT2 genes [13,39]. The highest expression level of $E S M Y B 7$ was observed in fruit that may accumulate the greatest PA level. $E S M Y B 10$ had high similarity with ESMYB7, but it was not expressed in fruit.

\section{Experimental Section}

\subsection{Plant Materials}

Tissues used in this study were sampled in March-April 2011 from the Hunan population of E. sagittatum originally transplanted from Hunan province, China in 2006 and grown in the experimental field of Wuhan Botanical Garden, Wuhan, China up to now. Young leaf, young petiole, flower, root and fruit tissues were harvested at the blooming stage except fruits, immediately frozen in liquid nitrogen and stored at $-70{ }^{\circ} \mathrm{C}$ until required.

\subsection{DNA and RNA Extraction}

Total genomic DNA was extracted from young leaves using the CTAB (cetyltrimethylammonium bromide) method [45]. Total RNA was isolated from fully expanded red leaves using TRIzol reagent (Invitrogen, Carlsbad, CA, USA). The RNA solution was digested with RQ1 RNase-Free DNase (Promega, Madison, WI, USA) to remove any DNA contamination before the reverse transcription reaction.

\subsection{Reverse Transcription and Isolation of R2R3-MYB cDNA Clones}

In this study, a total of $13 R 2 R 3-M Y B$ cDNA clones were isolated from red young leaves, of which four cDNA clones (designated ESMYB1 to EsMYB4) were obtained using homology-based cloning method as described below, with the remaining nine cDNA clones (designated EsMYB5 to EsMYB13) isolated from the leaf EST database of E. sagittatum [25]. Specifically, total RNA (1 $\mu \mathrm{g})$ from red leaves was reverse transcribed using oligo(dT) ${ }_{18}$ and Superscript II reverse transcriptase (Invitrogen, Carlsbad, CA, USA) and the reaction product was diluted 10-fold for use as cDNA template for MYB clone isolation. Four pairs of degenerate primers for EsMYB1 to EsMYB4 (Table S4) were designed to amplify the MYB domain and then the corresponding full-length cDNA clones were amplified with the SMART RACE Amplification kit (Clontech, Mountain View, CA, USA). In other way, the MYB domain was blasted against the E. sagittatum EST database and nine R2R3-MYB full length cDNA clones were finally obtained using the RACE technology. All gene specific primers for isolation of full-length cDNA clones and the corresponding genomic DNA clones are listed in Table S4. PrimeSTAR HS DNA Polymerase (Takara, Dalian, Liaoning, China) was used to guarantee high fidelity of the sequence. The full-length cDNA sequences of 13 R2R3-MYB genes from E. sagittatum 
have been deposited in the GenBank database (accession no. JN426948-JN426960 for EsMYB1 to EsMYB13).

\subsection{Sequence and Phylogenetic Analysis}

Blast program [46] was used to determine candidate genes and ORF finder program [47] was used to search open reading frames (ORF). Spidey program [48] was used to analyze the exon and intron genomic structure and the Conserved Domain program [47] was used to search conserved domains for Epimedium MYB genes. Multi-alignment analysis was performed using ClustalW [49] and MEGA 4.0 [40] was used to generate a neighbor-joining phylogenetic tree. Default parameters were used for all programs unless otherwise mentioned.

\subsection{Quantitative RT-PCR}

Quantitative RT-PCR was carried out to detect the expression patterns of 13 isolated R2R3-MYB genes in different tissues of E. sagittatum. Total RNAs from leaf, petiole, flower and root were extracted with TRIzol reagent (Invitrogen, Carlsbad, CA, USA), while total RNA from fruits was isolated using RNAiso Plus and RNAiso-mate for Plant Tissue kits (Takara, Dalian, Liaoning, China). One microgram of total RNA was reverse transcribed with a Primescript RT reagent kit and gDNA eraser (Takara, Dalian, Liaoning, China) was used to remove any contaminated genomic DNA. The quantitative PCR (qPCR) assay was undertaken according to manufacturer's instructions using the SYBR Premix Ex Taq II kit (Takara, Dalian, Liaoning, China) and run on an ABI7500 Real-Time PCR machine (Applied Biosystems, Foster, CA, USA). Gene specific primers for qPCR assay were designed using the Primer Premier 5 software and the Actin homolog was used as an internal control to normalize the expression level (Table S5). After the end of qPCR program, the melt curve was performed to ensure the amplification of the specific products. The comparative $\mathrm{Ct}$ method was used to determine the relative expression [50].

\subsection{Total Anthocyanin Content Determination}

Total anthocyanins were extracted and measured using spectrophotometric method as described by Mancinelli [51]. Red and green leaves were collected at the different developmental stages and frozen at $-70{ }^{\circ} \mathrm{C}$ until use. The samples (about 200-300 mg) were ground into fine powder with liquid nitrogen. Anthocyanins were extracted with $1 \% \mathrm{HCl}$ in methanol for $24 \mathrm{~h}$ at $4{ }^{\circ} \mathrm{C}$ in darkness with occasional shaking. The extracts were centrifuged and decanted carefully, and their absorbance was measured at $530 \mathrm{~nm}$ (peak absorption of anthocyanins) and $657 \mathrm{~nm}$ (peak absorption of chlorophyll degradation products). The equation A530 - $0.25 \times$ A657 was used to compensate for the absorption of chlorophyll degradation products at $530 \mathrm{~nm}$. Total anthocyanin content was showed as the subtracted absorbance/fresh weight. Three independent replicates were analyzed for each sample.

\section{Conclusions}

In summary, thirteen $R 2 R 3-M Y B$ genes from $E$. sagittatum were isolated and characterized through homology-based cloning and a systematic survey of EST database followed by full-length sequencing. 
Detailed sequence information for thirteen $R 2 R 3-M Y B$ genes provides a forward step into the molecular characterization of whole $R 2 R 3-M Y B$ family in Epimedium. Based on the results of sequence, phylogenetic and transcription expression analyses it is predicted that five $E s M Y B$ genes (ESMYB1, EsMYB7, EsMYB9, EsMYB10 and EsMYB12) are likely to be involved in the regulation of various branches of the phenylpropanoid biosynthetic pathway in E.sagittatum. The isolation and molecular characterization of $R 2 R 3-M Y B$ genes will facilitate the understanding of regulation of the flavonoid biosynthetic pathway and even the biosynthesis of the bioactive compounds in Epimedium. The five $E S M Y B$ genes could be selected as candidate genes for plant metabolic engineering studies in future to produce the desired compounds. Gain-of-function and loss-of-function experiments using transgenic plants are planned to verify the predicted biological functions of the different EsMYB genes.

\section{Acknowledgments}

This study was supported by grants from the National Natural Science Foundation of China (No. 31270340), and CAS/SAFEA International Partnership Program for Creative Research Teams Project and Knowledge Innovation Project of The Chinese Academy of Sciences (KSCX2-EW-J-20). We greatly thank Alice Hayward from University of Queensland for critical editing of the manuscript.

\section{Conflict of Interest}

The authors declare no conflict of interest.

\section{References}

1. Riechmann, J.L.; Heard, J.; Martin, G.; Reuber, L. Arabidopsis transcription factors: Genome-wide comparative analysis among eukaryotes. Science 2000, 290, 2105-2110.

2. Dubos, C.; Stracke, R.; Grotewold, E.; Weisshaar, B.; Martin, C.; Lepiniec, L. MYB transcription factors in Arabidopsis. Trends Plant Sci. 2010, 15, 573-581.

3. Lipsick, J.S. One billion years of Myb. Oncogene 1996, 13, 223-235.

4. Ogata, K.; Hojo, H.; Aimoto, S.; Nakai, T.; Nakamura, H.; Sarai, A.; Ishii, S.; Nishimura, Y. Solution structure of a DNA-binding unit of Myb: A helix-turn-helix-related motif with conserved tryptophans forming a hydrophobic core. Proc. Natl. Aca. Sci. USA 1992, 89, 6428-6432.

5. Stracke, R.; Werber, M.; Weisshaar, B. The R2R3-MYB gene family in Arabidopsis thaliana. Curr. Opin. Plant Biol. 2001, 4, 447-456.

6. Jiang, C.; Gu, X.; Peterson, T. Identification of conserved gene structures and carboxy-terminal motifs in the Myb gene family of Arabidopsis and Oryza sativa L. ssp. indica. Genome Biol. 2004, 5, R46.

7. Wilkins, O.; Nahal, H.; Foong, J.; Provart, N.J.; Campbell, M.M. Expansion and diversification of the Populus R2R3-MYB family of transcription factors. Plant Physiol. 2009, 149, 981-993.

8. Matus, J.T.; Aquea, F.; Arce-Johnson, P. Analysis of the grape MYB R2R3 subfamily reveals expanded wine quality-related clades and conserved gene structure organization across Vitis and Arabidopsis genomes. BMC Plant Biol. 2008, 8, 83. 
9. Deluc, L.; Bogs, J.; Walker, A.R.; Ferrier, T.; Decendit, A.; Merillon, J.M.; Robinson, S.P.; Barrieu, F. The transcription factor VvMYB5b contributes to the regulation of anthocyanin and proanthocyanidin biosynthesis in developing grape berries. Plant Physiol. 2008, 147, 2041-2053.

10. Jin, H.; Martin, C. Multifunctionality and diversity within the plant MYB-gene family. Plant Mol. Biol. 1999, 41, 577-585.

11. Paz-Ares, J.; Ghosal, D.; Wienand, U.; Peterson, P.; Saedler, H. The regulatory c1 locus of Zea mays encodes a protein with homology to myb proto-oncogene products and with structural similarities to transcriptional activators. EMBO J. 1987, 6, 3553-3558.

12. Hichri, I.; Barrieu, F.; Bogs, J.; Kappel, C.; Delrot, S.; Lauvergeat, V. Recent advances in the transcriptional regulation of the flavonoid biosynthetic pathway. J. Exp. Botany 2011, 62, 2465-2483.

13. Nesi, N.; Jond, C.; Debeaujon, I.; Caboche, M.; Lepiniec, L. The Arabidopsis TT2 gene encodes an R2R3 MYB domain protein that acts as a key determinant for proanthocyanidin accumulation in developing seed. Plant Cell Online 2001, 13, 2099-2114.

14. Borevitz, J.O.; Xia, Y.; Blount, J.; Dixon, R.A.; Lamb, C. Activation tagging identifies a conserved MYB regulator of phenylpropanoid biosynthesis. Plant Cell Online 2000, 12, 2383-2393.

15. Bogs, J.; Jaffé, F.W.; Takos, A.M.; Walker, A.R.; Robinson, S.P. The grapevine transcription factor VvMYBPA1 regulates proanthocyanidin synthesis during fruit development. Plant Physiol. 2007, 143, 1347-1361.

16. Czemmel, S.; Stracke, R.; Weisshaar, B.; Cordon, N.; Harris, N.N.; Walker, A.R.; Robinson, S.P.; Bogs, J. The grapevine R2R3-MYB transcription factor VvMYBF1 regulates flavonol synthesis in developing grape berries. Plant Physiol. 2009, 151, 1513-1530.

17. Jeong, S.T.; Goto-Yamamoto, N.; Hashizume, K.; Kobayashi, S.; Esaka, M. Expression of VvmybA1 gene and anthocyanin accumulation in various grape organs. Am. J. Enol. Viticulture 2006, 57, 507-510.

18. Gonzalez, A.; Zhao, M.; Leavitt, J.M.; Lloyd, A.M. Regulation of the anthocyanin biosynthetic pathway by the TTG1/bHLH/Myb transcriptional complex in Arabidopsis seedlings. Plant $J$. 2008, 53, 814-827.

19. Broun, P. Transcriptional control of flavonoid biosynthesis: A complex network of conserved regulators involved in multiple aspects of differentiation in Arabidopsis. Curr. Opin. Plant Biol. 2005, 8, 272-279.

20. Ramsay, N.A.; Glover, B.J. MYB-bHLH-WD40 protein complex and the evolution of cellular diversity. Trends Plant Sci. 2005, 10, 63-70.

21. Guo, B.; Xiao, P. Comment on main species of herba epimedii. China J. Chin. Mater. Medica 2003, 28, 303-307.

22. Ma, H.; He, X.; Yang, Y.; Li, M.; Hao, D.; Jia, Z. The genus Epimedium: An ethnopharmacological and phytochemical review. J. Ethnopharmacol. 2011, 134, 519-541.

23. Wu, H.; Lien, E.J.; Lien, L.L. Chemical and Pharmacological Investigations of Epimedium Species: A Survey. In Progress in Drug Research; Birkhäuser Verlag: Berlin, Germany, 2003; Vol. 60, pp. 1-57. 
24. Commission, P. Pharmacopoeia of the People's Republic of China. Chemical Industry Press: Beijing, China, 2005; Vol. 1, pp. 229-229.

25. Zeng, S.; Xiao, G.; Guo, J.; Fei, Z.; Xu, Y.; Roe, B.A.; Wang, Y. Development of a EST dataset and characterization of EST-SSRs in a traditional Chinese medicinal plant, Epimedium sagittatum (Sieb. Et Zucc.) Maxim. BMC Genomics 2010, 11, 94.

26. Shen, P.; Guo, B.; Gong, Y.; Hong, D.Y.Q.; Hong, Y.; Yong, E. Taxonomic, genetic, chemical and estrogenic characteristics of Epimedium species. Phytochemistry 2007, 68, 1448-1458.

27. Xie, P.S.; Yan, Y.Z.; Guo, B.L.; Lam, C.; Chui, S.; Yu, Q.X. Chemical pattern-aided classification to simplify the intricacy of morphological taxonomy of Epimedium species using chromatographic fingerprinting. J. Pharmaceutical Biomed. Anal. 2010, 52, 452-460.

28. Li, Z.; Xu, Y.; Wang, Y.; Huang, H. Status and prospect of research on medicinal plants of Epimedium L. Chin. Tradit. Herbal Drugs 2005, 36, 289-295.

29. Kranz, H.D.; Denekamp, M.; Greco, R.; Jin, H.; Leyva, A.; Meissner, R.C.; Petroni, K.; Urzainqui, A.; Bevan, M.; Martin, C. Towards functional characterisation of the members of the R2R3-MYB gene family from Arabidopsis thaliana. Plant J. 1998, 16, 263-276.

30. Jin, H.; Cominelli, E.; Bailey, P.; Parr, A.; Mehrtens, F.; Jones, J.; Tonelli, C.; Weisshaar, B.; Martin, C. Transcriptional repression by AtMYB4 controls production of UV-protecting sunscreens in Arabidopsis. EMBO J. 2000, 19, 6150-6161.

31. Terrier, N.; Torregrosa, L.; Ageorges, A.; Vialet, S.; Verries, C.; Cheynier, V.; Romieu, C. Ectopic expression of VvMybPA2 promotes proanthocyanidin biosynthesis in grapevine and suggests additional targets in the pathway. Plant Physiol. 2009, 149, 1028-1041.

32. Galego, L.; Almeida, J. Role of DIVARICATA in the control of dorsoventral asymmetry in Antirrhinum flowers. Genes Dev. 2002, 16, 880-891.

33. Howarth, D.G.; Donoghue, M.J. Duplications and expression of DIVARICATA-like genes in Dipsacales. Mol. Biol. Evolut. 2009, 26, 1245-1258.

34. Zimmermann, I.M.; Heim, M.A.; Weisshaar, B.; Uhrig, J.F. Comprehensive identification of Arabidopsis thaliana MYB transcription factors interacting with R/B like BHLH proteins. Plant J. 2004, 40, 22-34.

35. Deluc, L.; Barrieu, F.; Marchive, C.; Lauvergeat, V.; Decendit, A.; Richard, T.; Carde, J.P.; Mérillon, J.M.; Hamdi, S. Characterization of a grapevine $R 2 R 3-M Y B$ transcription factor that regulates the phenylpropanoid pathway. Plant Physiol. 2006, 140, 499-511.

36. Zhou, X.R.; Wang, Y.Z.; Smith, J.F.; Chen, R. Altered expression patterns of TCP and MYB genes relating to the floral developmental transition from initial zygomorphy to actinomorphy in Bournea (Gesneriaceae). New Phytol. 2008, 178, 532-543.

37. Tamagnone, L.; Merida, A.; Parr, A.; Mackay, S.; Culianez-Macia, F.A.; Roberts, K.; Martin, C. The AmMYB308 and AmMYB330 transcription factors from Antirrhinum regulate phenylpropanoid and lignin biosynthesis in transgenic tobacco. Plant Cell Online 1998, 10, 135-154.

38. Aharoni, A.; de Vos, C.; Wein, M.; Sun, Z.; Greco, R.; Kroon, A.; Mol, J.N.M.; O’Connell, A.P. The strawberry FaMYB1 transcription factor suppresses anthocyanin and flavonol accumulation in transgenic tobacco. Plant J. 2001, 28, 319-332. 
39. Yoshida, K.; Iwasaka, R.; Kaneko, T.; Sato, S.; Tabata, S.; Sakuta, M. Functional differentiation of Lotus japonicus TT2s, R2R3-MYB transcription factors comprising a multigene family. Plant Cell Physiol. 2008, 49, 157-169.

40. Tamura, K.; Dudley, J.; Nei, M.; Kumar, S. MEGA4: Molecular evolutionary genetics analysis (MEGA) software version 4.0. Mol. Biol. Evolution 2007, 24, 1596-1599.

41. Pérez-Rodríguez, P.; Riaño-Pachón, D.M.; Corrêa, L.G.G.; Rensing, S.A.; Kersten, B.; Mueller-Roeber, B. PlnTFDB: Updated content and new features of the plant transcription factor database. Nucleic Acids Res. 2010, 38, D822-D827.

42. Grotewold, E.; Sainz, M.B.; Tagliani, L.; Hernandez, J.M.; Bowen, B.; Chandler, V.L. Identification of the residues in the Myb domain of maize $\mathrm{C} 1$ that specify the interaction with the bHLH cofactor R. Proc. Natl. Aca. Sci. USA 2000, 97, 13579-13584.

43. Yanhui, C.; Xiaoyuan, Y.; Kun, H.; Meihua, L.; Jigang, L.; Zhaofeng, G.; Zhiqiang, L.; Yunfei, Z.; Xiaoxiao, W.; Xiaoming, Q. The MYB transcription factor superfamily of Arabidopsis: Expression analysis and phylogenetic comparison with the rice MYB family. Plant Mol. Biol. 2006, 60, 107-124.

44. Legay, S.; Lacombe, E.; Goicoechea, M.; Brière, C.; Séguin, A.; Mackay, J.; Grima-Pettenati, J. Molecular characterization of EgMYB1, a putative transcriptional repressor of the lignin biosynthetic pathway. Plant Sci. 2007, 173, 542-549.

45. Doyle, J.; Doyle, J. Isolation of plant DNA from fresh tissue. Focus 1990, 12, 13-15.

46. Altschul, S.F.; Gish, W.; Miller, W.; Myers, E.W.; Lipman, D.J. Basic local alignment search tool. J. Mol. Biol. 1990, 215, 403-410.

47. Wheeler, D.L.; Church, D.M.; Federhen, S.; Lash, A.E.; Madden, T.L.; Pontius, J.U.; Schuler, G.D.; Schriml, L.M.; Sequeira, E.; Tatusova, T.A.; et al. Database resources of the National Center for Biotechnology. Nucleic Acids Res. 2003, 31, 28-33.

48. Wheelan, S.J.; Church, D.M.; Ostell, J.M. Spidey: A tool for mRNA-to-genomic alignments. Genome Res. 2001, 11, 1952-1957.

49. Larkin, M.A.; Blackshields, G.; Brown, N.P.; Chenna, R.; McGettigan, P.A.; McWilliam, H.; Valentin, F.; Wallace, I.M.; Wilm, A.; Lopez, R.; Thompson, J.D.; Gibson, T.J.; Higgins, D.G. Clustal W and Clustal X version 2.0. Bioinformatics 2007, 23, 2947-2948.

50. Livak, K.J.; Schmittgen, T.D. Analysis of relative gene expression data using real-time quantitative PCR and the $2^{-\Delta \Delta \mathrm{C}_{\mathrm{T}}}$ method. Methods 2001, 25, 402-408.

51. Mancinelli, A. Interaction between light quality and light quantity in the photoregulation of anthocyanin production. Plant Physiol. 1990, 92, 1191-1195.

(C) 2013 by the authors; licensee MDPI, Basel, Switzerland. This article is an open access article distributed under the terms and conditions of the Creative Commons Attribution license (http://creativecommons.org/licenses/by/3.0/). 